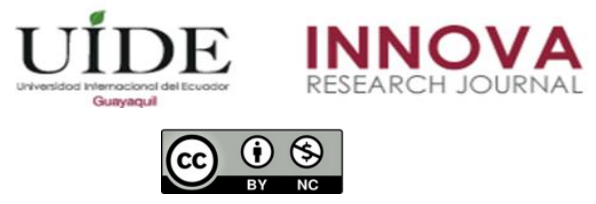

INNOVA Research Journal, ISSN 2477-9024

(Septiembre-Diciembre 2020). Vol. 5, No.3 pp. 182-195

DOI: https://doi.org/10.33890/innova.v5.n3.2020.1401

URL: http://revistas.uide.edu.ec/index.php/innova/index

Correo: innova@uide.edu.ec

\title{
Grupos Focales: Marco de Referencia para su Implementación
}

\section{Focus Groups: A Framework for Implementation}

Fabián Darío Rodas Pacheco

https://orcid.org/0000-0001-8263-5015

Universidad de Cuenca, Ecuador

Vicente Guillermo Pacheco Salazar

https://orcid.org/0000-0001-8409-6967

Universidad de Cuenca, Ecuador

Autor para correspondencia: fabian.rodas@ucuenca.edu.ec; guillermo.pacheco@ucuenca.edu.ec

Fecha de recepción: 22 de abril del 2020 - Fecha de aceptación: 07 de agosto del 2020

\section{Resumen}

La técnica de grupos focales ha sido utilizada por los investigadores en las ciencias sociales y comportamentales por más de ochenta años. Sin embargo, debido a la amplia gama de información respecto de grupos focales, se consideró pertinente la necesidad de tener una guía clara para la ejecución de esta técnica en el área social. En este escenario, el objetivo de la presente revisión de la literatura es definir los principios teóricos y criterios esenciales respecto de los grupos focales para proveer, tanto a estudiantes como a investigadores, los insumos necesarios requeridos para la aplicación de esta técnica, al momento de recabar datos. Este estudio se enmarca en la metodología de tipo de revisión bibliográfico descriptivo. En este contexto, se observa que los términos método y técnica son usados como sinónimos, siendo estos diferentes en acepción, que no hay consenso en el número de preguntas a formular durante la entrevista, y que el número de participantes debería constar de seis a diez colaboradores.

Palabras Clave: grupos focales; entrevista; metodología cualitativa; investigación; participantes

\begin{abstract}
The focus group technique has been used by researchers in social and behavioral sciences for more than eighty years. However, amid a wide spectrum of information regarding focus groups, it is pertinent the need to have clear guidelines for its implementation in the social scope. In this scenario, the objective of this literature review aims at defining the theoretical principles and essential criteria regarding the focus groups to provide both students and researchers with the necessary tools required for the implementation of this technique, at the time of collecting data. This study is part of the descriptive bibliographic review type methodology. In this context, it is observed that the terms method and technique are used as synonyms, these being different in meaning, there is no consensus on the number of questions asked during the interview, and the number of participants should consist of six to ten collaborators.
\end{abstract}

Key Words: focus groups; interview; qualitative methodology; research; participants 


\section{Introducción}

Los grupos focales, como una herramienta para recabar información cualitativa, se ha convertido en una alternativa válida muy utilizada como instrumento de recolección de datos cualitativos en diversas áreas del conocimiento en las últimas décadas (Rabiee, 2004). En este contexto, esta revisión literaria presenta cinco momentos sobre los grupos focales: antecedentes históricos, aproximaciones conceptuales, criterios para su aplicación, análisis de los datos y bondades y limitaciones. Con el propósito de entender la razón de los grupos focales, es importante analizar su trayectoria en el tiempo, por lo tanto, se ha llevado a cabo un breve análisis histórico. A continuación, se ha realizado una revisión de conceptos con el propósito de aterrizar en una noción clara para tener una mejor comprensión de lo que es un grupo focal. Respecto de los criterios, se consideran ciertas características de los grupos focales entre las cuales constan una guía para la entrevista, la forma de selección de los participantes, la duración de la entrevista, el número de participantes y las características del moderador. Este trabajo concluye presentando recomendaciones para el análisis de los datos y las bondades y limitaciones de la implementación de la técnica de grupos focales.

\section{Métodos}

Durante la aplicación de grupos focales para estudios doctorales, se observó la carencia de información base que provea al investigador de un sustento teórico claro y de pasos concretos a seguir para la implementación de esta técnica al momento de recolectar datos. Guirao Goris (2015) menciona que "la rápida progresión del conocimiento científico mediado por la aparición creciente de publicaciones en un medio como la internet requiere la realización de revisiones de la literatura científica que nos permitan tener un conocimiento actualizado sobre un tema de interés" (p. 1). Por consiguiente, surge la necesidad de trabajar en una revisión de la literatura con el objetivo de recolectar información de varias fuentes respecto de grupos focales para aterrizar en un documento guía que indique objetivamente qué es un grupo focal y cuáles son sus implicaciones al momento de su aplicación. En este marco, es importante comprender que la revisión de varias fuentes permite al investigador diagnosticar e identificar qué se conoce y qué se desconoce respecto de éste.

Este estudio se enmarca en la metodología de tipo de revisión bibliográfico descriptivo que según Day (2005) "proporciona al lector una puesta al día sobre conceptos útiles en áreas en constante evolución. Este tipo de revisiones tiene una gran utilidad en la enseñanza y también interesará a muchas personas de campos conexos" (p. 11). Para realizar esta revisión bibliográfica descriptiva se consultaron alrededor de sesenta y cinco insumos como artículos científicos, libros, sitios web oficiales, entre otros. También se tomó como referencia a Hart (2018), quién recomienda enfocarse en ocho preguntas clave para tamizar objetivamente la información perteneciente al tema; sin embargo, seis han sido usadas debido a la especificidad del estudio: ¿Cuáles son las teorías, conceptos e ideas clave en el tema? ¿Cuáles son las fuentes clave? ¿Cuáles son las cuestiones principales y las controversias sobre el tema? ¿Cuál es el origen y la definición del tema? Y ¿Cómo se estructura y se organiza el conocimiento sobre el tema? 


\section{Antecedentes históricos}

Los métodos de grupos focales se convirtieron en los pilares fundamentales de transmisión ancha, mercadeo e investigación sobre opinión pública luego de la Segunda Guerra Mundial, pero también fueron altamente ignorados en investigación y evaluación académica hasta finales de los años 70 (Merton R. K., 1987).

A lo largo de la historia se observa que investigadores que han trabajado con grupos en disciplinas básicas y aplicadas como administración, antropología, ciencias de la salud, ciencias políticas, comunicación, educación, gerontología, ingeniería en sistemas, marketing, psicología social, psicoterapia y sociología, han utilizado la técnica de grupo focal como una fuente confiable para la recolección de información primaria (Stewart \& Shamdasani, 2015).

Kidd y Parshall (2000) mencionan que el método de los grupos focales se desarrolló a partir de los métodos de investigación diseñados por Paul Lazarsfeld, Robert Merton y sus colegas en el Departamento de Investigación Social Aplicada de la Universidad de Columbia, Estados Unidos, con el propósito de evaluar las respuestas a propaganda y a transmisiones de radio durante la Segunda Guerra Mundial. Por su parte, Lazarsfeld había ideado un sistema electrónico para la grabación cuantitativa de reacciones tanto positivas como negativas de la audiencia, por lo que invitó a Merton para que colabore en el desarrollo de mejores métodos de obtención de la información (Merton, Fiske, \& Kendall, 1990).

Este método, originalmente conocido como entrevista focal (Merton R.K., 1987), fue utilizado tanto para entrevistas grupales como individuales, siendo más comunes las grupales. En este contexto, Merton y sus colegas manifestaron que las entrevistas grupales podían liberar o incrementar el nivel de inhibición en los participantes, dependiendo del tema y de la composición del grupo (Kidd \& Parshall, 2000).

Merton et al. (1990) consideraban que ciertas ventajas de la entrevista grupal eran, por ejemplo, una mayor cantidad de respuestas y obtención de algunos detalles que de otra manera podrían ser pasados por alto. Por otro lado, entre las desventajas se destacaban la desviación a temas irrelevantes y la competencia entre los miembros del grupo por dominar la entrevista. Adicionalmente, Kidd y Parshall (2000) dan a conocer que el aspecto más sobresaliente de las entrevistas focales, en contraposición con métodos más convencionales de investigación que utilizaban entrevistas, constaba el hecho de que todos los entrevistados se destacaban por haber experimentado, según Merton y Kendall (1946), algún tipo de situación concreta y particular, la que se convirtió en el foco de la entrevista. Asimismo, los investigadores habían realizado un tipo de análisis a priori de aquella situación focal con el objetivo de desarrollar una guía de entrevista para obtener información relevante sobre las preguntas de investigación e hipótesis (Kidd \& Parshall, 2000). En esta misma línea, estos autores sostienen que la idea no era elaborar una entrevista altamente estructurada. Al contrario, se debía trabajar con preguntas que motivasen a los miembros del grupo a expresarse libremente; esto daba lugar a que, al enfocarse en preguntas simples sobre aspectos específicos de un programa, producto o evento, era posible ir más allá de una mera interpretación de lo que estaba bien o de aquello que era erróneamente percibido. 


\section{Aproximaciones conceptuales}

Escobar y Bonilla-Jiménez (2017) sostienen que los grupos focales provienen de diferentes posturas epistemológicas, como el realismo y el constructivismo contextual. En el realismo los datos son analizados desde categorías preexistentes, buscando la consistencia del significado entre múltiples análisis y aplicando los conceptos de confiabilidad y validez. Por su parte, el constructivismo contextual considera que no existe una sola realidad que pueda ser revelada a través de la correcta aplicación de un método. Estos autores concluyen que el conocimiento es temporal y dependiente del contexto; por lo tanto, refuta los criterios de objetividad y la confiabilidad a favor de estrategias de reflexión y articulación por parte del investigador. (Escobar \& Bonilla-Jimenez, 2017).

Powell, Single, y Loyd (1996) definen al grupo focal como "un grupo de individuos seleccionados y reunidos por el investigador con el propósito de discutir y comentar, desde su experiencia, sobre un tema que es el objeto de estudio de la investigación" (p. 499).

Adicionalmente, Thomas, MacMillan, McColl, Hale, y Bond (1995) sostienen que un grupo focal es una técnica que involucra una entrevista a profundidad en la que los participantes son seleccionados a propósito, conformando una muestra de una población específica con el objetivo de enfocarse en un tema dado.

Martínez Reyes (2012) manifiesta que, "aunque más conocida como grupo focal, esta técnica también recibe los nombres de entrevista de grupo focal, grupo de discusión, discusión de grupo y se le conoce en inglés como focus group o group interview" (p. 47). Adicionalmente, este autor sostiene que es una técnica de recolección de datos de tipo cualitativo ubicada dentro de la entrevista, pero con carácter grupal que se usa dentro de las ciencias sociales.

Por su parte, Morgan (1997) indica que la información que se obtiene de un grupo focal proviene de la interacción de los participantes y es en esencia el interés del investigador lo que provee el foco. Asimismo, Dörnyei (2007) resalta que las entrevistas a grupos focales son algunas veces consideradas como una subclase de entrevistas debido a que el formato utilizado en el rol del entrevistador es diferente al del rol de una entrevista individual. En este contexto, un grupo focal requiere de un formato especial en donde el entrevistador graba las respuestas de un grupo pequeño, usualmente conformado por 6 a 12 miembros.

Por su parte, Edmunds (1999) menciona que los grupos focales dan lugar a que el investigador capte los comentarios subjetivos, para luego evaluarlos, buscando de esta manera proveer un entendimiento de las percepciones, los sentimientos, las actitudes y las motivaciones de los miembros del grupo.

Para Martinez-Migueléz (1999), el grupo focal es el método de investigación colectivista, más que individualista, que se centra en la pluralidad y variedad de las actitudes, experiencias y creencias de los participantes, y lo hace en un espacio de tiempo relativamente corto. Adicionalmente, Dörnyei (2007) indica que este método constituye una forma económica de juntar una gran cantidad de información cualitativa y luego utilizarla para una variedad de propósitos en diferentes áreas, pues se basa en la experiencia colectiva, lo que permite que los participantes se inspiren, cuestionen y reaccionen frente a situaciones emergentes. Bertoldi, 
Fiorilo, y Alvarez (2006), por su parte, consideran que un grupo focal usualmente está constituido por un conjunto de individuos quienes se convierten en los informantes. En este contexto, el investigador primero los selecciona y los organiza alrededor de un tema. Luego coordina el proceso de interacción, discusión y elaboración de acuerdos en un mismo espacio y en un tiempo definido. En este mismo escenario, Edmunds (1999) recomienda utilizar grupos focales cuando el concepto o tema que se pretende evaluar es relativamente nuevo y se requiere obtener ideas creativas. Asimismo, para Krueger y Casey (2000), el grupo focal busca un espectro de ideas y sentimientos que los individuos del grupo tienen sobre ciertos temas. Además, el propósito es discutir situaciones que influyan en opiniones, conductas y motivaciones. Por lo tanto, si se desea que surjan ideas del grupo, éste tiene la capacidad de convertirse en más que la suma de sus partes, pues tiene la ventaja de dar a conocer una energía que las personas cuando están solas no pueden. Según Rabiee (2004), los grupos focales proveen una amplia gama de ideas y sentimientos que los individuos tienen sobre cierto tema, a la vez que resaltan las diferencias en las perspectivas de los miembros del grupo. Adicionalmente, Green, Draper, y Dowler (2003) recuerdan que lo singular del grupo focal es su habilidad para generar datos basados en la sinergia de la interacción grupal.

Hamui-Sutton y Varela-Ruiz (2013) establecen que los grupos focales tienen la bondad de recolectar información respecto de sentimientos, pensamientos y vivencias de los participantes, provocando auto explicaciones con el propósito de obtener datos cualitativos. Finalmente, Nyumba, Wilson, Derrick, y Mukherjee (2018) indican que el grupo focal es una técnica utilizada para entender problemas sociales a profundidad.

\section{Criterios para su aplicación}

Nyumba et al. (2018) establecen que la técnica de grupo focal tiene como objetivo obtener datos de un grupo de individuos deliberadamente seleccionados y de forma presencial. Sin embargo, McDermott (2013) menciona que también es factible aplicar esta técnica a través de webcams, entornos virtuales y redes sociales.

\section{Guía para la entrevista}

La entrevista es la etapa fundamental del grupo focal, ya que de allí sale la información cualitativa. Por lo tanto, es importante tener una idea clara del número de preguntas a hacer, el tipo de preguntas y consideraciones respecto de cómo llevar a cabo la entrevista grupal. Morgan (1997) resalta que el objetivo de una guía es recabar información significativa respecto del tema investigado. En este escenario, Merton et al. (1990) enfatizan cuatro puntos importantes para la entrevista grupal. Primero, la entrevista debería incluir un grupo de preguntas que son relevantes al tema. Segundo, ésta debería recoger información específica sobre lo tratado. Tercero, la entrevista debería promover la interacción que permita examinar los sentimientos de los participantes con cierta profundidad. Cuarto, tomar nota del contexto personal descrito durante la entrevista, pues a menudo los participantes no están conscientes de sus propias perspectivas hasta que interactúan con otros. Por su parte, Redmond y Curtis (2009) añaden que el objetivo de un grupo focal es crear un ambiente en donde salgan a la luz una gran variedad de perspectivas. De esta manera, la guía se convierte en una herramienta que permite que la información fluya a lo largo de la entrevista, de inicio a fin (Vaughn, Shay Schumm, \& Sinagub, 1996) 
Redmond y Curtis (2009) indican que la estructura de un diálogo en un grupo focal dependerá del tipo de entrevista que se va a aplicar. Morgan (1997), por su parte, manifiesta que uno de los tipos de entrevista es la estructurada. Esta requiere de cuatro a cinco preguntas que se llevan a cabo presencialmente.

Krueger y Casey (2002) sostienen que el conjunto de preguntas se conoce como "questioning route" que en español significa una "hoja de ruta". Estos autores recomiendan que se debería elaborar alrededor de doce preguntas para un tiempo aproximado de dos horas. Adicionalmente, sugieren tener en cuenta tres pasos a seguir para la elaboración de estas preguntas: i. realizar una lluvia de ideas respecto del tema a investigar, ii. elaborar un borrador de las preguntas y iii. validar las preguntas a través de la técnica de revisión.

Aigneren (2002) y Krueger (2006) coinciden en que el número de preguntas debe mantenerse entre cinco y seis y que estas tienen que ser de tipo abiertas.

Krueger y Casey (2002) indican que las preguntas deben ser realizadas de manera exacta como fueron redactadas. Stewart y Shamdasani (2015) y Escobar y Bonilla-Jimenez (2017) concuerdan en que una buena guía de entrevista consta de preguntas simples, sin ambigüedad, que guíen a los participantes y que deberían partir de preguntas generales a preguntas más específicas.

\section{Participantes y su selección}

Redmond y Curtis (2009) recuerdan que se utilizan los grupos focales cuando se requiere recabar información específica de personas con ciertas características y con un conocimiento similar sobre un tema particular. En este sentido, Krueger (1990) y Litosseliti (2003) mencionan que los individuos que conforman un grupo se sienten más libres de expresar sus puntos de vista cuando notan que los otros miembros son similares a ellos; por consiguiente, las características de los participantes de un grupo focal estarían definidas por el propósito de la investigación, el que considera factores biológicos como la edad, sexo, antecedentes educativos y conocimientos y experiencia sobre el tema tratado (Redmond \& Curtis, 2009). Sobre este mismo tema, Vaughn et al. (1996) comentan que las variables más comunes al momento de seleccionar a los participantes de un grupo focal son edad, sexo, raza y clase social.

Adicionalmente, Stewart y Shamdasani (2015) comentan que aquellos individuos que han sido seleccionados para participar en una investigación deberían estar dispuestos y preparados para proveer la información requerida. Morgan (1997), por su parte, sugiere que los participantes de un grupo focal deberían ser elegidos utilizando una muestra intencional. Escobar y BonillaJimenez (2017) sostienen que los participantes pueden provenir de grupos homogéneos o heterogéneos, dependiendo del estudio. Sin embargo, estos autores recomiendan tener un grupo homogéneo si el estudio tiene como objetivo recabar información que provenga de experiencias compartidas. Finalmente, Talbot (1995) y Polit y Tatano Beck (2006) proponen a los investigadores seleccionar a los participantes de un grupo focal tomando en cuenta su conocimiento y experiencia con respecto al tema a investigar. 
Bloor, Frankland, Thomas, y Robson (2001) indican que la interacción entre los participantes constituye un elemento clave al momento de conformar los grupos focales. Por su parte, Redmond y Curtis (2009) añaden que cuando se selecciona a los participantes, es fundamental asegurarse que cada miembro esté dispuesto a colaborar. Asimismo, es necesario que los participantes se sientan cómodos interactuando entre ellos; por consiguiente, se debe evitar que exista una gran disparidad con respecto a sus antecedentes sociales. En este escenario, Morgan (1997), recuerda que, si bien el objetivo de un grupo focal es la homogeneidad, ésta tiene que ver con la homogeneidad en bagaje cultural y no actitudinal. Finalmente, Redmond y Curtis (2009) recuerdan que es el investigador quien tiene que tomar una serie de decisiones clave basadas en los objetivos de su investigación.

\section{Duración de la entrevista}

Con respecto a la entrevista, esto dependerá de la complejidad del tema investigado y del número de preguntas, pues podría durar entre una y dos horas (Redmond \& Curtis, 2009). En este mismo escenario, Gibson (2007) sugiere que los adolescentes pueden mantener su atención no más de cuarenta y cinco minutos a una hora; mientras que los jóvenes adultos, con un moderador experimentado, la atención y fluidez en la discusión se puede mantener hasta noventa minutos. Krueger y Casey (2000) advierten que dos horas es el límite físico y psicológico de las personas y que la entrevista con un grupo focal no debería prolongarse más allá de ese tiempo. Kuhn (2018) menciona que normalmente un grupo focal puede durar entre una y dos horas, enfatizando en las ventajas y desventajas que estos dos rangos de tiempo podrían tener. Este autor sostiene que la ventaja de tener un grupo focal de sesenta minutos es que en una tarde o en un día el investigador puede abarcar la aplicación de un mayor número de grupos focales, incluyendo una gran variedad de participantes. Por otra parte, indica que dos horas es el tiempo promedio ya que permite obtener información relevante al estudio y a los objetivos de investigación. Finalmente, recomienda que el tiempo de un grupo focal se mantenga en noventa minutos por tres razones: i. permite acoger a más grupos en una sola tarde, optimizando el tiempo de aplicación. ii. permite al investigador tener el tiempo suficiente para crear el ambiente apropiado para la aplicación del grupo focal. Y iii. permite a los participantes mantenerse enfocados en la discusión, evitando la fatiga.

\section{Número de participantes}

La literatura menciona que existen varios pronunciamientos con respecto al número óptimo de participantes en un grupo focal. Por un lado, Morgan (1997) y Bloor et al. (2001) comentan que algunos autores sugieren conformar los grupos con seis hasta diez participantes. Por otro lado, Pugsley (2018) reporta que ciertos investigadores han utilizado desde tres hasta catorce individuos. En todo caso, el número ideal de participantes dependerá del tema a tratarse y del conocimiento que estos tengan de este. Morgan (1997) recomienda conformar el grupo focal con diez o doce miembros, mientras que Krueger y Casey (2000) recomiendan trabajar con seis hasta ocho participantes con el propósito de que el moderador pueda manejar la discusión.

Kuhn (2018) menciona que una de las ventajas de tener un grupo pequeño (cuatro-seis) es que se elimina el cansancio que se experimenta en grupos más grandes, evitando la fatiga de los participantes. 
Dörnyei (2007) por su parte indica que el tamaño de un grupo focal varía de entre seis y diez participantes, y en algunos casos hasta doce. Sin embargo, indica que, de tener menos de seis participantes, se limitaría el pensamiento colectivo y se dificultaría la participación individual.

Finalmente, la literatura recomienda tener de entre seis a diez participantes por grupo. Sin embargo, Escobar y Bonilla-Jimenez (2017) sugieren que "el tamaño del grupo focal también depende del nivel de profundidad que se le va a dar a la entrevista por parte del investigador y de los objetivos de la investigación."

\section{El moderador}

Redmond y Curtis (2009) manifiestan que la entrevista de un grupo focal es conducida por un facilitador conocido como moderador. Zavaleta (2016) indica que el moderador "dirige el diálogo basado en una guía de entrevista previamente elaborada, da la palabra a los participantes y busca estimular su participación equitativa" (p. 3). Por su parte, Stewart et al. (2007) sostienen que el moderador es una persona clave al momento de recabar información válida del grupo. Hamui-Sutton y Varela-Ruiz (2013) mencionan que es importante que el moderador cuente con una guía de entrevista y que determine los acuerdos previos a la aplicación del grupo focal, aclarando los objetivos y las instrucciones a tomarse en cuenta durante la entrevista. Por ejemplo, quién realiza las preguntas y quién las contesta.

Karger (1987), Hamui-Sutton y Varela-Ruiz (2013) y Zavaleta (2016) han recolectado información respecto de las características principales que debe tener en cuenta un moderador para llevar a cabo un grupo focal de manera eficiente. A continuación, se condesan estas características en la siguiente tabla:

Tabla. 1

Características para la aplicación de un grupo focal

\begin{tabular}{ll}
\hline & Involucrar sutilmente a los participantes en la discusión. \\
Motivar hábilmente a que interactúen entre ellos para lograr una sinergia óptima. \\
Permitir que la sesión fluya naturalmente. \\
Manejar el silencio adecuadamente. \\
Permanecer sin poses autoritarias y sin ningún tipo de prejuicios con respecto a lo que \\
manifiestan los participantes del grupo focal.
\end{tabular}

\begin{tabular}{ll}
\hline & Conocer el tema que va a indagar. \\
& Dar especificidad a las situaciones referidas. \\
& Mantenerse abierto a cualquier novedad que surja en el proceso y explorarla. \\
& Cuidar las relaciones interpersonales durante la conversación. \\
Hamui-Sutton y & Tener claridad y sencillez de expresión lingüística. \\
Varela-Ruiz (2013) & Ser amable y mostrar sensibilidad de escuchar con atención. \\
& Dar confianza a los participantes, tomando en cuenta lo que dicen y absteniéndose de \\
& dar su opinión. \\
\hline \multirow{2}{*}{ Zavaleta (2016) } & Mantener una mentalidad abierta a explorar cualquier novedad que surja en el \\
& proceso. \\
& Cuidar las relaciones interpersonales entre los participantes durante la entrevista. \\
\hline
\end{tabular}

Fuente: elaboración propia 
Zavaleta (2016) manifiesta incluir a un relator durante el grupo focal con el objetivo de llevar una bitácora de la información que se proporcione durante la sesión, a pesar de que esta sea grabada. Estos datos complementan el análisis y son de gran importancia ya que podría haber información que el moderador no logre captar.

Finalmente, Hamui-Sutton y Varela-Ruiz (2013) sugieren que el moderador debe agradecer a los participantes por su valiosa colaboración y respaldar los datos recolectados durante una sesión, para a posteriori realizar la respectiva trascripción.

\section{Análisis de los datos}

La investigación cualitativa y, en particular las entrevistas a grupos focales, genera una gran cantidad de información, que resulta abrumadora no sólo para los investigadores novatos sino también para los experimentados, pues una hora de entrevista usualmente toma de cuatro a cinco horas para transcribirla y genera de treinta a cuarenta hojas (Rabiee, 2004). Por su parte, Krueger y Casey (2000) mencionan que el propósito del análisis es canalizar los datos recolectados, concentrándose en el objetivo principal. Asimismo, Rabiee (2004) considera que, a pesar de que la mayor fuente de análisis de los datos proviene del lenguaje hablado grabado, las reflexiones sobre la entrevista, el lugar donde se realiza y el registro de la comunicación no verbal expresada por los miembros del grupo, aportan significativamente en la construcción y análisis de la información. Adicionalmente, este autor manifiesta que la forma de registrar la información se la puede realizar a través de la grabación de un audio o un video, acompañado de un diario, en donde el moderador o facilitador anota sus observaciones, las que deberían ser registradas inmediatamente después de la entrevista.

El proceso de análisis de los datos cualitativos es una de las etapas más importantes de la investigación ya que de allí se obtiene la información para responder las preguntas de investigación basadas en datos confiables. En este marco, es clave tener presente diferentes tipos de software y métodos para llevar a cabo un análisis objetivo de la información recabada. Una revisión de la literatura muestra que los tipos de software y métodos más utilizados son: NVivo, ATLAS.ti, análisis temático, y teoría fundamentada.

\section{ATLAS.ti}

ATLAS.ti es un software propio que permite al investigador conocer y codificar sistemáticamente y a profundidad los datos recolectados, a través de la exploración de información con el editor de red, la herramienta de búsqueda de texto, nubes y tablas de frecuencia de palabras. ATLAS.ti cuenta con su versión 8 que es la más reciente y está disponible para Windows y Mac. No obstante, la versión 7 continúa siendo ampliamente utilizada (ATLAS.ti, 2012).

\section{NVivo}

NVivo es un software propio diseñado principalmente para el análisis de datos cualitativos a través de la organización y gestión de información que proviene de entrevistas, 
encuestas y datos de artículos científicos, con el objetivo de encontrar perspectivas en el estudio. Al momento NVivo cuenta con su última versión que es la 12; sin embargo, la versión 11 está disponible para Windows y Mac en un periodo de prueba gratuita de catorce días (NVivo, s.f.).

\section{Análisis Temático}

El método de análisis temático (AT) permite al investigador examinar datos cualitativos, provenientes de varias áreas del conocimiento para encontrar patrones de significados y dar respuesta a las preguntas de investigación planteadas en un estudio mixto o cualitativo. Existen seis fases para llevar a cabo un análisis temático: i. familiarización con la información recolectada. ii. generación de códigos. iii. generación de temas potenciales. iv. delimitación de temas. v. definición y categorización de temas. vi. redacción del análisis (The University of Auckland, s.f.).

\section{Teoría Fundamentada}

La teoría fundamentada es uno de los métodos más utilizados en la investigación cualitativa (Dörnyei, 2007).

La teoría fundamentada es un método de investigación naturalista que se utiliza principalmente para generar teoría. El investigador comienza con una consulta amplia en un área temática particular y luego recopila información relevante sobre el tema. A medida que continúan los procesos de acción de recopilación de datos, cada información se revisa, compara y contrasta con otra. A partir de este proceso de comparación constante, los puntos en común y las diferencias entre las categorías de información se vuelven claros y, en última instancia, se desarrolla inductivamente una teoría que explica las observaciones. Por lo tanto, las consultas que serán respondidas a través de la teoría fundamentada no se relacionan con dominios específicos sino con la estructura de cómo el investigador quiere organizar los hallazgos. (Glaser \& Strauss, 1999, p. 3)

\section{Codificación}

Coffey y Atkinson (1996) indican que es importante que el investigador codifique los datos con el objetivo de que la información recabada de las entrevistas de los grupos focales vaya tomando forma y respondiendo a las preguntas de investigación. Sin embargo, no existe un proceso determinado para la codificación de información en la etapa del análisis de datos.

DeCuir-Gunby, Marshall, y McCulloch (2011) recomiendan codificar los datos que provienen de: la información de la revisión de la teoría (theory-driven), la información de los datos recolectados (data-driven) y de los objetivos de la investigación (structural) que derivan del análisis de los datos primarios.

Corbin y Strauss (2014), sostienen que existen dos niveles principales de codificación: codificación abierta y codificación axial. DeCuir-Gunby et al. (2011) explican que la codificación abierta permite explorar los datos primarios para crear códigos y conceptos. Estos 
autores recomiendan usar esta codificación al inicio de la sistematización de los datos provenientes de las entrevistas de los grupos focales. Por otra parte, una vez que los códigos han sido creados, es necesario proceder con la codificación axial, permitiendo al investigador identificar cualquier tipo de conexión que pudiese existir entre los códigos creados en el proceso inicial.

\section{Bondades y limitaciones de los grupos focales}

Acocella (2012) indica que la técnica de grupo focal ha sido recientemente redescubierta por investigadores del área social y que en la actualidad se ha convertido en una técnica de investigación innovadora. En este escenario, es importante mencionar las bondades y limitaciones que los investigadores podrían afrontar durante su implementación.

\section{Bondades}

Dörnyei (2007) sostiene que los grupos focales son altamente flexibles ya que son utilizados en una gran variedad de áreas del conocimiento, por ejemplo, en el contexto educativo, estos son usados para evaluar el currículo y verificar la efectividad de un curso en particular con el propósito de comprender que es lo que no está funcionando y por qué. En este contexto, el autor recuerda que una de las ventajas de emplear grupos focales consiste en que las personas usualmente aceptan participar ya que consideran las sesiones agradables y estimulantes, obteniendo información valiosa. Adicionalmente, debido a que los grupos focales son versátiles y permiten obtener información más profunda, son a menudo utilizados en investigación mixta. También Stokes y Bergin (2006) indican que la aplicación de esta técnica no conlleva un gasto económico significativo para los investigadores. Finalmente, esta técnica permite generar ideas que son valiosas en la construcción de cuestionarios y de entrevistas a profundidad para la obtención de datos en varias áreas del conocimiento.

\section{Limitaciones}

Dörnyei (2007) recuerda que las limitaciones de los grupos focales están relacionadas con el hecho de que las entrevistas requieren de mucha preparación antes de implementarlas. Asimismo, el moderador precisa llevar a cabo una serie de funciones simultáneamente como: grabar, realizar las preguntas, filmar, asegurase de que todos participen, entre otras. Por su parte, Smithson (2000) menciona dos posibles limitaciones de los grupos focales: la primera tiene que ver con la tendencia a que sólo ciertas opiniones que son socialmente aceptadas emerjan y la segunda está relacionada con el hecho de que algunos participantes tiendan a dominar la entrevista. Adicionalmente, Acocella (2012) sostiene que una posible limitación sería el silencio del participante como una estrategia defensiva, proveniente del estrés de estar en un grupo. Finalmente, Dörnyei (2007) manifiesta que una entrevista focal puede ser difícil de transcribir ya que involucra a varias personas y muchas veces es complicado identificar qué participante tomó la palabra y en qué momento. Para evitar este inconveniente, el autor indica que ciertos investigadores recomiendan filmar la entrevista. 


\section{Recomendaciones y Conclusiones}

Se finaliza esta revisión sistemática de la literatura presentando recomendaciones y conclusiones respecto de la implementación de los grupos focales.

Con respecto al número y al tipo de preguntas, se sugiere que sean relevantes al tema y que provean la interacción necesaria para examinar a profundidad las experiencias de los participantes. En este contexto, la recomendación general es que este set de preguntas se alinee a los objetivos de la investigación.

El rol del moderador es trascendental en el desarrollo de la técnica de grupo focal, ya que es el encargado de crear el ambiente propicio para la obtención de la información por parte de los participantes. También es importante recalcar que la aplicación de un grupo focal demanda logística y tiempo, ya que su implementación normalmente requiere de 30 a 60 minutos. En este escenario, es necesario utilizar varios recursos para la grabación de la entrevista con el propósito de respaldar los datos recopilados durante esta sesión.

A lo largo de la revisión de la literatura se observó que algunos autores utilizaban la palabra técnica, mientras que otros utilizaban el término método para referirse a un grupo focal. Estos términos fueron a menudo utilizados como sinónimos; sin embargo, existe una diferencia entre ellos. La técnica es el conjunto de recursos utilizados para alcanzar un objetivo, mientras que el método es el proceso que se sigue al momento de obtener una meta. En este marco, se considera que el método es más abarcador y la técnica es parte de este método. En el caso concreto de grupos focales, estos serían en realidad una técnica ya que requieren de ciertos lineamientos y recursos para su ejecución.

Finalmente, existen varias posiciones respecto del número de participantes dentro de un grupo focal. Los estudios dan a conocer números que varían de acuerdo al tipo de investigación y al entorno, concluyendo que el número promedio estaría entre seis a diez participantes.

\section{Bibliografía}

Acocella, I. (2012). The focus groups in social research: advantages and disadvantages. Quality \& Quantity, 46(4), 1125-1136.

Aigneren, M. (2002). La técnica de recolección de información mediante grupos focales. $L a$ Sociología en sus escenarios, (6).

ATLAS.ti. (2012). What is ATLAS.ti? Scientific Software Development GmbH. Retrieved from https://atlasti.com/product/what-is-atlas-ti/

Bertoldi, S., Fiorilo, M., \& Alvarez, M. (2006). Grupo focal y desarrollo local: Propuestas Para una articulación teórico metodológica. Ciencia, Docencia y Tecnología: $N^{\circ} 33,111-131$.

Bloor, M., Frankland, J., Thomas, M., \& Robson, K. (2001). Focus Groups in Social Research. London: Sage Publications.

Coffey, A., \& Atkinson, P. (1996). Making sense of qualitative data: complementary research strategies. Sage Publications, Inc.

Corbin, J., \& Strauss, A. (2014). Basics of qualitative research: Techniques and procedures for developing grounded theory. Sage publications. 
Day, R. A. (2005). Cómo escribir y publicar trabajos científicos. Pan American Health Org.

DeCuir-Gunby, J., Marshall, P., \& McCulloch, A. (2011). Developing and using a codebook for the analysis of interview data: An example from a professional development research project. Field methods, 23(2), 136-155.

Dörnyei, Z. (2007). Research Methods in Applied Linguistics: quantitative, qualitative, and mixed methodologies. Oxford University Press.

Edmunds, H. (1999). The focus group: Research handbook. Chicago, NTC Business Book. American Marketing Association.

Escobar, J., \& Bonilla-Jimenez, F. (2017). Grupos focales: una guía conceptual y metodológica. Cuadernos hispanoamericanos de psicología, 9(1), 51-67.

Gibson, F. (2007). Conducting focus groups with children and young people: strategies for success. Journal of research in nursing, 12(5), 473-483.

Glaser, B., \& Strauss, A. (1999). The Discovery of Grounded Theory: strategies for qualitative research.

Green, J., Draper, A., \& Dowler, E. (2003). Short cuts to safety: risk and 'rules of thumb' in accounts of food choice. Health, Risk and Society 5, 33-52.

Guirao Goris, S. J. (2015). Utilidad y tipos de revisión de literatura. Scielo, 0-0.

Hamui-Sutton, A., \& Varela-Ruiz, M. (2013). La técnica de grupos focales. Investigación en educación médica, 2(5), 55-60.

Hart, C. (2018). Doing a literature review: Releasing the research imagination. Sage.

Karger, T. (1987). Focus groups are for focusing, and for little else. Marketing News. (21), 52-55.

Kidd, P., \& Parshall, M. (2000). Getting the Focus and the Group: Enhancing Analytical Rigor in Focus Group Research. Sage Journals, 293-308.

Krueger, R. (1990). Focus Groups. A Practical Guide for Applied Research. London: Sage Publications.

Krueger, R. (2006). Is it a focus group? Tips on how to tell. Journal of Wound Ostomy \& Continence Nursing, 33(4), 363-366.

Krueger, R., \& Casey, M. (2000). Focus Groups: A Practical Guide for Applied Research. Third Edition. London: Sage Publications.

Krueger, R., \& CaseY, M. (2002). Designing and conducting focus group interviews.

Kuhn, G. (2018). What is the Best Length for a Focus Group? Retrieved from https://www.driveresearch.com/market-research-company-blog/what-is-the-best-lengthfor-a-focus-group-qualitative-research-firm/

Litosseliti, L. (2003). Using Focus Groups in Research. London: Continuum.

Martínez Reyes, N. (2012). Reseña metodológica sobre los grupos focales. Editorial Universidad Don Bosco. $N^{\circ}$ 9, 47-53.

Martinez-Migueléz, M. (1999). La investigación cualitativa etnográfica en educación. México: Trillas.

McDermott, M. (2013). Take your pick. ANA Magazine, 32-42.

Merton, R. K. (1987). The Focussed Interview and Focus Groups: Continuities and Discontinuities. The Public opinion quarterly 51.4, 550-566.

Merton, R., \& Kendall, P. (1946). The Focused Interview. American Journal of Sociology, 95.

Merton, R., Fiske, M., \& Kendall, P. (1990). The focused interview (2nd ed.). New York: Free Press.

Morgan, L. (1997). Focus groups as qualitative research. Sage publications. 
NVivo. (n.d.). What is NVivo. QSR International Pty Ltd. Retrieved from https://www.qsrinternational.com/nvivo/what-is-nvivo

Nyumba, T., Wilson, K., Derrick, C., \& Mukherjee, N. (2018). The use of focus group discussion methodology: Insights from two decades of application in conservation. Methods in Ecology and evolution, 9(1), 20-32.

Polit, D., \& Tatano Beck, C. (2006). Essentials of Nursing Reserach: Methods, Appraisal, and Utilization. Philadelphia,PA: Lippincott Williams and Wilkins.

Powell, R., Single, H., \& Loyd, K. (1996). Focus Groups in Mental Health Research: Enhancing the Validity of User and Provider Questionnaires. International Journal of Social Psychiatry. 42(3), 193-206.

Pugsley, L. (2018). Focus groups, young people and sex education. In Gender and Qualitative Research (1996) (pp. 114-130). Routledge.

Rabiee, F. (2004). Focus-group interview and data anlysis. Proceedings of the Nutrition Society, (pp. 655-660).

Redmond, R., \& Curtis, E. (2009). Focus groups: principles and process. Nurse researcher, 16(3).

Smithson, J. (2000). Using and analysing focus groups: limitations and possibilities. International Journal of Social Research Methodology. 3(2), 103-119.

Stewart, D., \& Shamdasani, P. (2015). Focus groups: Theory and practice. Sage publications.

Stokes , D., \& Bergin, R. (2006). Methodology or "methodolatry"? An evaluation of focus groups and depth interviews. Qualitative market research: An international Journal.

Talbot, L. (1995). Principles and Practice of Nursing Research. St. Louis MO: Mosby.

The University of Auckland. (n.d.). Thematic Analysis: a reflexive approach. Retrieved from https://www.psych.auckland.ac.nz/en/about/thematic-analysis.html

Thomas, L., MacMillan, J., McColl, E., Hale, C., \& Bond, S. (1995). Comparison of focus group and individual interview methodology in examining patient satisfaction with nursing care. Social Sciences in Health 1, 206-219.

Vaughn, S., Shay Schumm, J., \& Sinagub, J. (1996). Focus Group Interviews in Education and Psychology. Thousand Oaks, Ca.: Sage Publications.

Zavaleta, J. (2016). Los grupos focales como estrategia para recolectar información. Retrieved from http://www.espolea.org/uploads/8/7/2/7/8727772/ddt-gruposfocales.pdf 